\title{
INTRODUCTION OTD-NET AND LAS: ORDER-TO-DELIVERY NETWORK SIMULATION AND DECISION SUPPORT SYSTEMS IN COMPLEX PRODUCTION AND LOGISTICS NETWORKS
}

\author{
Klaus Liebler \\ Ulrike Beissert \\ Marco Motta \\ Axel Wagenitz \\ Fraunhofer Institute Material Flow and Logistics (Fhg IML) \\ Joseph-von-Fraunhofer Straße 2-4 \\ 44227 Dortmund, GERMANY
}

\begin{abstract}
Global Sourcing adds to a company's value by realizing the best prices on a global market and making new markets accessible. However, increasing global orientation of the companies is associated with new challenges in planning and controlling the network processes to obtain a high service level and a guaranteed availability of supply goods. Order-to-Delivery Network simulation (OTD-NET) offers a simulation based approach for gaining insight in global networks. The OTD-NET suite provides services for modelling, simulating and analyzing complete logistic networks including the specifics of the different network participants like suppliers or producers. With the specialized automotive edition, OTD-NET enables a precise specification of complex products considering its configuration, bill of materials and supports an easy analysis of various network strategies like network design, structure and process concepts, supply strategies as well as different production strategies. OTD-NET is developed by Fraunhofer Institute for Material Flow and Logistics in Dortmund (Germany). Within the paper OTD-NET is presented and exemplarily demonstrated by a joint project with Volkswagen.
\end{abstract}

\section{INTRODUCTION}

During the last decade, global sourcing, production and sales networks as well as associated intercontinental logistics relations have become increasingly important, and numerous studies show that this trend will continue (e.g., Cohen et al. 2008). Nowadays, in nearly all branches, the market demands innovative, customized and cost-effective products with high quality and short delivery time. Thus, companies face major challenges regarding their efficiency in production and logistics caused by global orientation and demand-oriented production. For example, it is necessary to distribute the customers' demand over the global production network in such a way that the available capacities are equally balanced and highly utilized. Simultaneously, a cost-optimized production should be provided while ensuring the service objectives such as delivery time, delivery reliability and availability. Besides these challenges, an increasing global orientation is associated with new opportunities for the companies regarding economics feasibility e.g. global sourcing adds to company's value through realizing the best prices on a global market (Motta et al. 2008).

To meet the increased market needs, flexibility and dynamism is becoming essential for a company (Toth, Wagenitz, and Klingebiel 2011). Companies must now face the challenge to transform from a traditional supply chain towards to a strategically oriented value network. Thus, cost-effectiveness, efficien 


\section{Liebler, Beissert, Motta, and Wagenitz}

cy and competitiveness depend essentially on a company's ability to purposefully restructure existing processes and organizational structures based on a detailed evaluation of the current status and forecasts of future demands. Hence, it is becoming more and more important to simulate the company or network processes and to evaluate it under a variety of perspectives. A thorough approach to planning and control of value-adding processes across the entire value network is needed to remain competitive and expand existing benefits of a network structure (Arnold et al 2008). The objective of such an approach is

- continuous optimization of existing processes and structures through the analysis and evaluation of strategy, structure and process alternatives.

- constant improvement of relevant performance and cost characteristics of the production and logistics network like on-time delivery, reduction of stocks and thereby implied costs and shorter lead and delivery times.

Looking at the automotive industry, requirements for an adequate planning system to handle complex supply chain network can be derived. Automotive industry is characterized by a broad production network with various participants involved like original equipments manufacturer (OEM), suppliers as well as various logistic service providers to execute transports between the supply chain partners. The automotive industry is characterized by a high product variety; German premium brands in particular offer a wide variety of customer configurable options. Almost every aspect of the car's interior and exterior may be changed or adapted. Meyr (2004) states that cars are available in more than 1030 variants (Meyr 2004). Therefore the number of customer configured build-to-order (BTO) cars increases. On the other hand, the global markets are different. The European market typically requires highly configurable BTO cars and therefore accepts the required order lead times. The North American market, on the other hand, requires shorter lead times and does not use the opportunity to configure an individual car very often. The planning process and the procurement of needed parts when the customer orders a certain variant in a BTO production results in average lead times up to 10 weeks. In contrast to US customers, European customers are willing to wait that time for their cars if they are customized. Due to finite customer patience, during the planning process, automotive companies must have accurate forecasts to ensure short delivery time, with high delivery reliability and availability and a detailed knowledge about the order penetration point (OPP) to be able to react flexibly to customer needs (Klingebiel et al. 2011).

Certain requirements for an integrated planning system to handle complex supply chain networks can be derived from the considered case. A large number of relevant elements is necessary to specify the organizational structure of the network as well as the organization's strategies. All influencing parameters on the network have to be specified like customers and their behavior, trade and distribution units, order processes, real job data, product specifications and their bills of materials, suppliers of any number of stages in the network, production units and resources, distribution structures and cost factors for all elements. The optimal configuration of the processes in a value network and the creation of a cost-optimized structure are subject to various dynamic interactions. The required level of detail for design and evaluation process requires handling very large models and large amounts of data. Because these requirements push conventional simulators to their limits, simulation is usually not used in the planning processes, although the obvious benefits include gaining insight, considering stochastic influences and determining the dynamic behavior and interdependencies. The Fraunhofer Institute for Material Flow and Logistics (IML) provides with OTD-NET a proven tool suite for process design and evaluation. OTD-NET allows an integrated specification of intra- and inter-company processes, structures and strategies in value networks, to vary the network conditions and to evaluate it according to performance criteria and cost-benefit considerations. 


\section{CONCEPT OF OTD-NET}

\subsection{General Description}

Fraunhofer IML started development of the simulation tool OTD-NET in 1998, based on the ideas of application-oriented and component-based modeling of logistic networks, particularly for the automotive industry, implementing relevant practical knowledge of experts. Before development, the IML compared existing methods and concepts while considering the specific challenges of automotive production networks, its specific processes, and complex product structures. However, existing methods and tools showed significant disadvantages like no capability for describing customizable features and parts list of the product, very limited selection of development tools, and no data structures like hash tables and Btrees to efficiently handle the data volume (Wagenitz 2007).

To overcome these drawbacks, the OTD-NET tool, an event-driven simulation environment, is provided. OTD-NET integrates concepts from business process simulation, supply chain management, and material flow simulation to simulate extremely complex supply, production, distribution network structures, material flow, and information and planning processes (Wagenitz 2007). According to the VDI directive 3633 (VDI 1992), OTD-NET consists of four main components (cf. Figure 1):

1. OTD-simulator - simulator core component: OTD-NET is a discrete-event simulation tool for the simulation based analysis of supply networks. It allows detailed mapping and analysis of complex production networks with all their constraints as well as planning, execution and material flow processes based on an easily expandable object-oriented class-model and object-oriented relational databases. In the simulator core, "Real world" entities, informed by domain-specific knowledge, are represented by C++ objects and the class structure is documented using UML. Furthermore, each relevant $\mathrm{C}++$-class is accompanied by a GME-class, and GME objects are used to instantiate $\mathrm{C}++$-objects. However, OTD as simulation tool suite is generalizable to all industries because of its abstract object-oriented structures.

2. OTD-Analyzer - data management: OTD-Analyzer enables multi-dimensional OLAP- or SQLbased analysis of result data with provided chart designs. The analyzer evaluates the comprehensive results from the simulation core and summarizes key, understandable information from complex structures. The OTD-Analyzer allows the definition and analysis of individual KPIs (key performance indicators) based on different views (customer-specific, supplier-specific or plant-specific). Furthermore the analysis covers a wide range of characteristic KPIs and considers different cost factors like:

- Value stream, production KPIs and capacity loads

- Inventories and inventory coverage

- Transport frequencies, times and delivery reliabilities

- Capital commitment and inventory costs

- Transportation costs incl. duty and insurance if required

3. GME - Graphical Modelling Environment: The GME enables the detailed description and modelling of all available simulation objects. For example, complex products can be modeled considering constraints, prohibitions and property groups based on implemented supply network objects and related shapes. Bill of materials (BOM) in the product description can calculate required components for assembly by disintegration of the final product. OTD-NET can quickly model complex tasks with applicationoriented and abstract component categories. The application-oriented category involves components for mapping processes, structures, resources and products with corresponding domain knowledge. For example, components for mapping the product structure provide the conventional views of the automotive industry with families of customer-selectable properties, equipment packages, as well as constraints and prohibitions. Other components are available in the library to model the production and logistics processes like plants, suppliers and distribution channels. Different views or scenarios can be modeled by using the provided parameters of the objects without any additional programming, like shift models for plants or transport characteristics such as timetables for distribution channels. On the other hand, for modeling 
special use cases and highly individual processes, the simulation engineer can apply many non-prespecified abstract elements.

4. Interfaces: OTD-NET aims to also provide simulation technologies for the operational phase, which must represent the actual condition of the network with all current assets, capacities, transports, etc. OTD-NET provides numerous interfaces for automated model generation, integrating data from the operational systems of network entities like enterprise resource planning systems (ERP) of factories, warehouse management systems (WMS) in storage locations or transport management systems (TMS) from logistics service providers

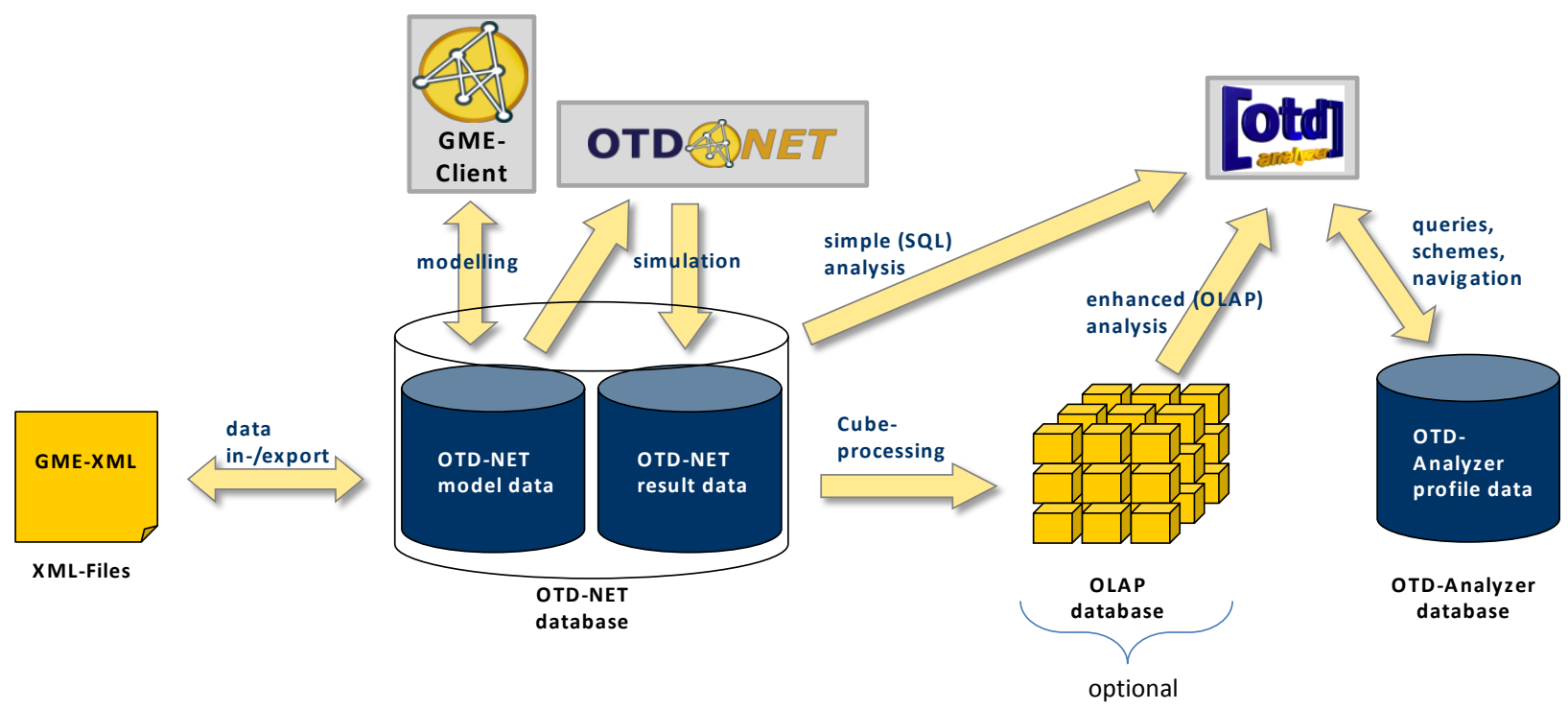

Figure 1: Components of OTD-NET and interaction

The simulation model tracks and logs each part on each process, resulting in gigabytes of raw data managed by OTD-NET's database management which can be analyzed efficiently with OLAP. It is possible to simulate multi-stage networks over several years in a few minutes on standard PCs.

\subsection{Modeling in OTD-NET}

As described above, OTD-NET is an object oriented simulation tool suite. The tool suite provides the GME for manual modeling, which makes the pre-defined objects accessible to the simulation engineer. An unique feature of OTD-NET is the integration of comprehensive operational knowledge in the simulation objects. The simulation engineer does not need to program the behavior of the objects, he only has to specify them by setting the provided object parameters. For showing the scope and the characteristics of the simulation objects, major elements are presented in detail.

\subsubsection{Product Configuration}

A distinctive feature of automotive products is the high variety of properties a customer can select. Within the mass customization of products, the OEM provides numerous technical and marketing variants of product properties. The available property combinations that can be selected by the customer are subject to technical and marketing requirements. All these requirements that determine the flexibility of selectable combinations are also of course time dependent. To meet the requirements for the model building of these product structures OTD-NET provides the following simulation objects: 


\section{Liebler, Beissert, Motta, and Wagenitz}

Product classes and variants: The product class is the root object for the product description. Product classes in OTD-NET can be hierarchically modeled to build up product variants. The product class contains descriptions for the variant determining properties which are element of all products and the customer selectable properties. All properties of the product variant itself and demand rules can be modeled as time dependent. The hierarchical product structure allows the inheritance of parameter settings and property specifications. Beside the technical product descriptions, cost aspects can be modeled in the product structure. For each product and each property, single and common costs related to purchasing, production, distribution, administration and marketing can be modeled as well as the revenue in different markets. The properties of the products are organized in property families. The embedded operational knowledge in the simulation allows the generation of valid products by checking the rules for manufacturing feasibility and ensuring that all required properties are elements of the product.

Property family and properties: Properties represent customer-selectable options. The different properties are grouped into property families. A property family includes all variants of properties for a certain component of the product. E. g. all available variants of headlights for a car are grouped within the property family headlights. This approach combines the basic equipment of the product with the customer-selectable options. By ensuring that one property from each property group is an element of the final product, only valid products are generated by the simulation core component.

Restrictions: The complex product "car" requires rules for the combination of the available properties and customer selectable options. These restrictions result from technical constraints (e. g. a case for sunglasses in the ceiling cannot be combined with a sun roof) and from marketing considerations (e. g. simple radio configurations are not available in a market with high revenues). Such restrictions can be modeled in OTD-NET with several allocations to markets, products, properties and time dependencies.

The modeled product structures are used by the simulator for the generation of valid product orders. In case of importing existing orders the simulator uses this information to verify the input data.

\subsubsection{Bill of Material}

OTD-NET provides the ability to model BOM rules. These BOM rules link a production location with a component, an amount and a boolean rule over the properties. The rule is constructed in postfix notation. This allows an easy linear storage in memory or in a database. Furthermore, the evaluation may be implemented very fast in a pushdown automata. Additionally, each rule may be equipped with a validity interval. If an order is scheduled for production in this interval at the given factory and its option set fulfills the rule, then the component demand occurs. BOM rules are referenced from the product descriptions in the hierarchical tree. For each order, the only BOM rules that have to be checked are those that are referenced on any ancestor node of the orders variant.

\subsubsection{Plants and Suppliers}

Within the modeled production networks, the plant-object assembles the final products. Required parts and components are delivered by the modeled suppliers. The network structure is finalized by modeling the distribution channels between suppliers, plants and dealers. All the mentioned simulation objects include various operative knowledge. The plant and supplier objects themselves include shift models, working times and production lead times. If required, even the build of a production sequence can be influenced by a car sequencing approach using Ho-No rules (Boysen 2007). The supply relations can be modeled by referencing suppliers for certain parts or components to the plant object. In case of dual or multi sourcing strategies, individual priorities for the suppliers can be modeled. The supplier objects are divided into BTO and BTS suppliers. The implemented business logic of the simulation core component proofs available capacities of BTO suppliers before booking an order to the production program of the plant. For the determination of required parts and components a BOM-explosion is carried out by the simulation core component. For modeling the logistic network between supplier, plants, and dealers, dis- 
tribution channels are provided as simulation objects. These include several parameters for influencing the behavior of the transport processes like schedules, on demand transports, transport lead times, loading lead times, etc. Therefore, for example, a truck transport or a scheduled railway transport can be modeled using these simulation objects. For complex logistic structures, rule-based routing can occur at any stage of the network. Rules can be modeled using all available attributes of the transported items.

All mentioned objects or production and transportation include cost parameters for single and common costs as described for the product structure objects.

\subsection{Logistic Assistance Systems (LAS)}

Simulation offers various benefits for planning logistics and production in global networks. Beside these planning capabilities, it is nowadays mainly used to carry out studies. To make the benefits of simulation available during operations the idea of Logistic Assistance Systems (LAS) was developed. Implementing LAS can eliminate problems arising from the advanced nature of OTD-NET and its difficult implementation in operational management. The LAS concept focuses on enhanced collaboration, consistent information over the whole supply chain, transparency about the current process status, planning functionality and fast system development and implementation. Basically, LAS are planning and software systems that can be adapted to new supply chain or planning situations with less effort and integrated into a company's organization very easily. Building up on the OTD-NET Framework, LAS support planning experts in recurring decision situations by making all relevant information of a supply chain transparent and integrating specific decision support systems (OTD-NET) as well as planning approaches into one combined planning approach (Toth, Wagenitz, and Klingebiel 2011). Thus, LAS assist planners to quickly identify critical situations and objectively evaluate consequences of possible decision alternatives.

For reaching maximum transparency in the entire supply chain, LAS integrate the information available in different operational systems like ERP, WMS, TMS, etc. from the focused part of the supply chain. Flexible interfaces are provided for the information integration. Developing the data integration logic is a key step in building a new LAS. By integrating data from different systems and logically transforming all this data, LAS can offer a single point of truth. All planners using the LAS share the same database and can use the LAS as an integrated and holistic communication platform.

In decision situation the planner often cannot foresee all the holistic consequences of his decision. Planning tools like simulation, especially based on OTD-NET models, are integrated into the LAS for forecasting decision effects. The LAS framework logic automatically applied the decision of the planner to the simulation model, masking the complexity of the simulation model. In the LAS user interface a domain specific language, the "planners language", for describing the action is used and translated to the accompanied model changes. The simulation itself proofs the consequences of the decision in a holistic way. Results of the simulated forecast are provided in the LAS and help the planner to make the right decision. Once a proofed action is approved to be applied, this information can be handed over by the LAS to the operational systems. These actions on the network can be monitored by updating the network data to gain insight.

The LAS framework is a Java based web application so all installation of simulation tools and databases occurs on the server side. Therefore, LAS is easily accessible without extraordinary hardware requirements on the client side. The web based architecture guarantees multi user accessibility which can be regulated, e. g. by using companywide subscription systems for a single sign on.

Toth at al. (2011) described that "LAS integrate functionalities of SCM-Systems like SCMo and SCEM. SCMo is a collaborative multi-level SCM concept that needs software support for processing information between network partners (Odette 2004). Their basic function is the exchange of production demands and inventory levels between business partners in supply networks to reach information transparency and avoid time lags in information flow.” (Toth, Wagenitz, and Klingebiel 2011) 
A better understanding of LAS features and power can be demonstrated with a practical example. Therefore, the following section describes an operational LAS from Volkswagen, used for ATP and CTP checks in the worldwide production network of the Volkswagen Amarok.

\section{USE CASE: SIMULATION IN LOGISTICS ASSISTANCE SYSTEMS FOR TACTICAL NETWORK PLANNING (VOLKSWAGEN ECO2LAS)}

The OTD-NET tool suite and derived LAS as shown above can be used for problems in the field of operational-tactical network planning. For this purpose, OTD-NET is embedded in a web-based assistance system. The functionality is not only accessible for a wide range of users, but also allows continuous monitoring of current and future material supply in complex production networks.

\subsection{Use Case}

Volkswagen Commercial Vehicles produces its light pickup truck "Amarok" at two sites in Hannover (Germany) and Pacheco (Argentina). Suppliers of parts as well as complex assemblies are located in Brazil and Argentina as well as in Europe. Both production sites receive material from suppliers on both continents. The multimodal transport methods are dominated by scheduled sea vessels between Brazil, Argentina and Germany. For faster delivery and to hedge against transportation delays, transport by air cargo is also possible. Container yards next to the production sites keep inventory for some days. This supply network is depicted schematically in Figure 2.

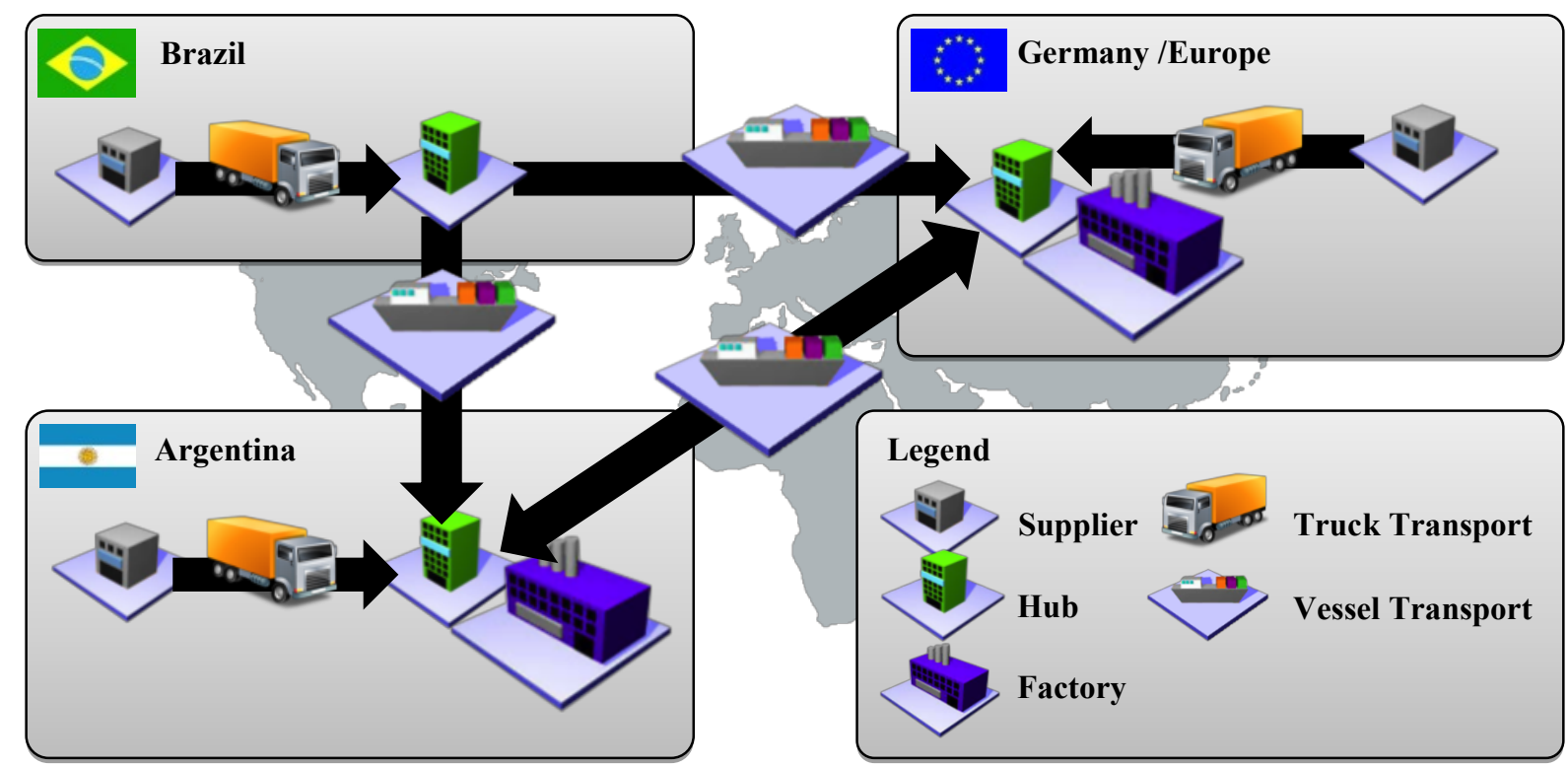

Figure 2: The "Amarok" Supply and Production Network

The following numbers depict the complexity of the "Amarok" supply and production network

- Permanently about 40,000 customer orders ready for production scheduling

- About 300 first tier suppliers

- 8,900 distinct material numbers

- Permanently 500 containers on transport with more than 42,000 different stock objects (combination of container * part number * amount)

The main runs executed by ship are scheduled to operate weekly. In the pre- and post-run sections, inland container vessels, trains or trucks are deployed to deliver on call. Whenever a bottleneck arises, air 
cargo would be dispatched as a contingency plan. Once containers arrive at the final production site, they are stored temporarily in a container yard. If contents are requested from production, a container is unpacked completely and all contents are transported to a warehouse near the production plant. Depending on the volume of goods, suppliers or consolidation centers pack material in containers in preparation for sea freight. Trucks transport the materials to the sea ports, arriving long before the ship's departure in order to have sufficient time to pass through customs and load material. The uncertainty about the time required for customs activities regularly provides dispatchers with major challenges. The customs checks may be delayed so much that an urgently needed container may be loaded on a later ship.

The complete lead time comprises the time for information and material flow from orders scheduling to material availability at the final assembly and is about 10 weeks long. Thereby, the time required for the material flow constitutes movement time, storage time and processing time.

Dispatchers at the production and suppliers sites are facing complex challenges regarding uncertainty of the material amount requested from overseas and guarantee a flexible production program at the sites in order to maintain order flexibility to the customer. Due to the fixed transport schedule both uncertainties may disturb the transport chain. A small disturbance in one stage of the multimodal transport chain may significantly impact all subsequent stages. Additionally, customers are now considering more environmental aspects not only limited to car's emissions and exhaust, but also the carbon footprint of production and distribution processes when deciding to purchase the final product. Hence, all processes and all changes in processes must to be evaluated with respect to both economic and environmental objectives.

\subsection{Objective and Solution}

To efficiently and holistically control the supply network described above, the car manufacturer need an integrated IT-system that provides:

- Transparency of current and future material flow

- Decision support for process efficiency and expected economic and ecologic cost of changing any measures

- Early identification of stock-out or overstock situations in near real-time

- Harmonization of material demand and material supply.

To fulfill these requirements, a Logistic Assistance System (LAS) was developed to support all planning tasks under economic and ecological objectives. Three functional modules constitute the LAS "eco2las" in this case:

- A Data Warehouse module with appropriate import filters (Farkisch 2011)

- The Methodical Core module embedded with OTD-NET

- A Web-based GUI module (see Figure 3). 


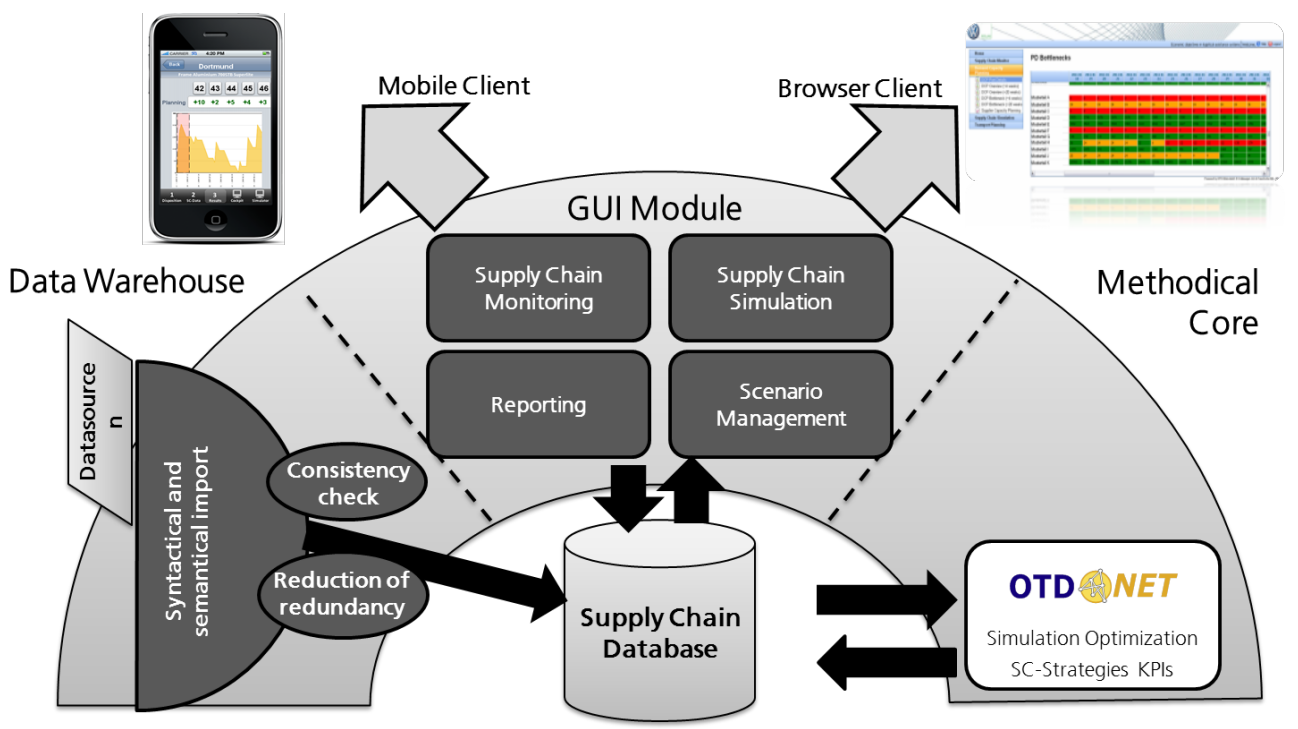

Figure 3: The architecture of "eco2las"

The data warehouse module enables storing of multiple versions of the relevant data for planning. Hence, the planning information can be updated daily automatically but can also be updated on-demand. Thereby, an ETL-Process (Extract-transform-load) is initiated, querying a large variety of data sources (e.g. operative IT systems, public databases, web services) and merging the data into an integrated and consistent model, the so called „master scenarios“. Users may also generate their own "user scenarios" from master-scenarios to conduct experiments.

The GUI-module provides user with access to all relevant data for planning, either directly available in the data warehouse or available after a simulation run. The module also allows users to easily change scenarios by an interface.

The simulation component of OTD-NET provides an dynamic and in-depth analysis of master- or user-scenarios. Therefore, all process steps from the acceptance of the customer order to delivery of the finished vehicle to the customer are created. A dedicated module in the LAS business layer turns the data over into the simulation component that models all relevant aspects of the supply chain and its management (cf. chapter 2.2). Furthermore, in the eco2las project, the following algorithmic modules of OTDNET (beyond standard simulation functionality) is used:

- rule-based BOM explosion

- planned orders generator

\subsection{Proceeding}

In short, the simulation develops the current supply network state towards a future state. The simulation begins from the point when the supply chain state is fetched from operative systems. Then, all warehouses in the simulation are pre-filled with the initial stock. All customer orders are injected into the order queue. Transports are put on their current position in the supply chain. After initialization, all stock levels are evolved from this state. Starting at the suppliers, new transports to the harbor are created and filled up with goods to known transport capacities. Ship transports follow their predefined schedule and on arrival, trigger the on-call transports from harbor to the production site. As transports arrive at the production site, the warehousing capacities fill up. The production of orders issues material demand calculation and appropriately reduces stock levels. This calculation of demand uses OTD-NET's high-performance rulebased BOM-explosion module, capable of applying hundreds of thousands of rules for millions of orders in less than one hour. 
The simulation results can be analyzed in appropriate views like dynamic course of stock levels (see Figure 4). This view of the final assembly line supports decision makers to determine if typical behavior of an unsynchronized supply chain should be expected, such as bottlenecks or overcapacities. Once an unsynchronized supply chain behavior is identified eco2las supports the generation of experimental userdefined scenarios based on the current master version. Thereby specific parameters of the model are changed. For example, if a shortage occurs in week 3, an expedited transport with an appropriate amount of parts may be injected into the model. The efficiency of this measure may be verified with a simulation run of OTD-NET. Beyond classic financial KPIs, eco2las provides detailed Carbon-dioxide calculation based on the European Life Cycle Database (ELCD, cf. (Institute for Environment and Sustainability 2010)). The method allows the consideration of ship schedules, capacity constraints of pre- and postcarriage and other dynamic restrictions. The decision maker ultimately chooses to implement the experiment with the lowest overall costs.

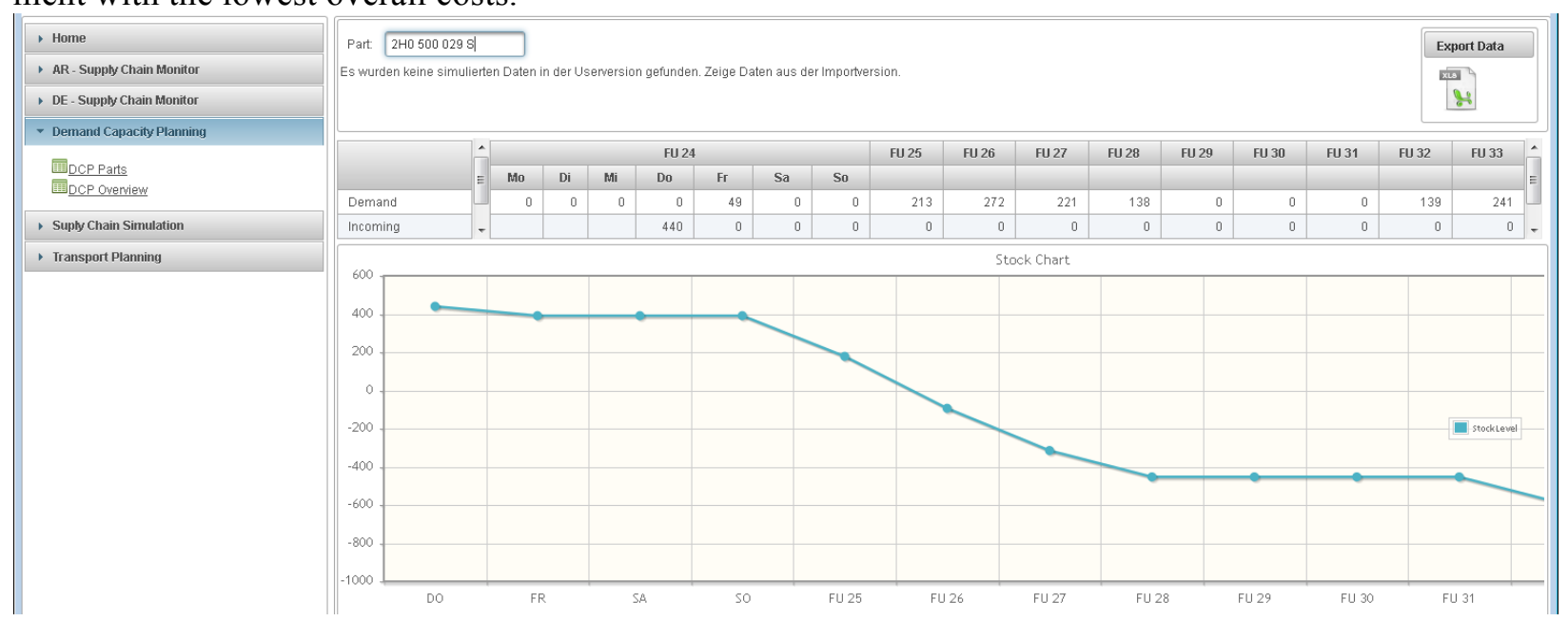

Figure 4: Simulation Results: Stock Level at the Hub next to the Factory in Hannover

The concept of Artificial Customer Orders facilitate the expansion of the planning horizon. Real customer orders are only available to fulfill production slots in the upcoming weeks. "Artificial customer orders" are calculated and implemented to extend the planning horizon beyond available real order data. The generating algorithm transforms abstract market predictions (volumes and take rates) into fully specified orders. These orders are consistent with the full product master data in that each car is valid according to option combination rules. Furthermore, the algorithm tries to fulfill the take rate predictions. Beyond that, a customer behavior model assures that co-correlated options are correctly represented (e.g. if a customer orders a navigation systems, it is probable, that he (as a business customer) also orders a hands free set).

\subsection{Evaluation}

The LAS system has reached production stability. Its unique features are leveraged to support the daily planning processes. The productivity of logistical planning is raised significantly. Formerly, dispatchers had to access fragmented information systems for questions over different time horizons, having to check multiple different systems for several supply chain sections separately for short- and mid-term questions. However, now all information is available in one system. All supply chain sections, from supplier orders to production order fulfillment, are monitored. Furthermore, the system covers both the short-term to midterm planning horizon with a homogeneous methodology. Decision makers have access to the full transparency of the material and information flows at all sections such that they can avoid stock outs. 


\section{Liebler, Beissert, Motta, and Wagenitz}

Additionally, with the customizability of user scenarios they may check the efficiency of countermeasures with the same means in minutes. An often used function is the "manage transports"-view. After having created their own "user scenario", users can modify transport times and check the consequences. The simulation can then be leveraged to answer the question, "Which parts will probably run out of stock, if an entire ship or a single container is delayed?". According to the user's changes, a new simulation model is constructed and a simulation run is conducted. This allows decision makers to freely and safely explore the dependencies of the supply chain. With OTD-NET they can then learn more about the behavior of individual relations in the network.

The simulation is currently being extended to even more realistically depict the supply chain process. Currently, the specific container-packing at the suppliers and consolidation centers is not addressed in the simulation. In simulation, all shipped goods are treated as if they were not packed. The complete containers may only be unpacked at the final destination, and then, all contents are transported towards assembly line. Consider a realistic case, in which a container has two different part types: one urgently requested part and one part which is already available in sufficient quantities. The forced full unload leads to a high level of "bycatch" - parts that are not needed, but provided due to process restrictions. To minimize this bycatch, a novel optimization method in OTD-NET is being developed and currently integrated. Here each cargo handling point in the supply chain will be assigned a specific time-to-line period, updated daily to reflect the current transport situation. This time is the sum of all transportation times and stock coverage times through the whole supply chain. The objective is to send as many batches of parts through the supply chain, on each section and at any time, such that the stock in the chain fulfills demand in the timeto-line period. This basic requirement is overlaid with an optimization procedure that seeks the favorable utilization of containers whilst considering physical parameters of packaging, weight and volume restrictions. As the existing simulation model provides all the data necessary for this algorithm, the method will be implemented as a new embedded planning module in OTD-NET. The core optimization algorithm combines a heuristic optimization method and a MILP. Preliminary results will be presented in a forthcoming paper.

\section{CONCLUSION AND OUTLOOK}

OTD-NET's unique modeling technique is able to recreate all essential aspects of an intercontinental automotive supply network. It provides an adequate balance between the effort required to model the system and the quality of results. Within this paper the added value of OTD-NET in planning and controlling network processes is presented by a project example for operational and tactical management. Nevertheless, OTD NET is also suited to answer questions in the tactical and strategic level.

The modeling elements are sophisticated and tailored to automotive supply chains. The entities in the simulation model incorporate a high level of expert knowledge. Hence, few parameters are necessary to define all essential aspects of the system, which then can perform a fast and full evaluation of intercontinental material flow. Models with high planning quality are constructible with minimal effort. The particular suitability for problems in the automotive industry can be underlined by the following modeling artifacts: modeling of installation and process structures, modelling of customized mass products, high performance algorithmic modules and add-on modules.

OTD-NET provides a configurable archetype for each element of a common automotive supply chain, making it easier to model installation and process structures in multistage supply networks. Only the behavior of the modeled transformation, storage, handling and transport sections may be adapted to the specific needs using the predefined behavior of the elements like forecasting, allocation, and call off strategies. These strategies are all recreated based on real aspects of the automotive industry and allow a realistic behavior of the overall model.

In addition to the installation and process structure, a realistic model of the simulation load has a significant influence on a realistic overall model. This load is represented in the short-term planning horizon by real customer orders and by sales forecasts in the medium-term planning horizon. As already shown, 
OTD-NET uses the property-based product description for variants (cf. chp. 2.2, Herlyn 1990). It has been shown in several projects that the modeling style of OTD-NET is manufacturer-independent and easily handles the complexity of the complete portfolio of German premium manufacturers.

High performance algorithmic modules facilitate the simulation of complex and high volume supply chains in minutes. A simulation run is typically conducted in less than five minutes. In combination with the fully automated simulation model construction, this allows for real-time permanent planning. All crucial algorithms are designed so that they can be executed multithreaded or even distributed, if necessary, by adding parallel computation units to significantly decrease processing times.

Add-on modules of OTD-NET can generate valid simulation models using information from PDMand ERP-systems for different manufacturers. Within a few minutes, planning-relevant information can be extracted from operational systems and be used to update or to construct a simulation model. This facilitates real-time realistic simulation.

OTD-NET is constantly evolving. Both the amount of modeling and the behavior of elements is expanded. A high degree of backward compatibility of the core components allows to check the simulation framework with predefined test cases and, hence, provide high quality software. Two recent developments include visualisation of the simulation in the web browser and the adoption of the modeling approaches in various industries with similar supply chains and product structures. These include, for example, agricultural machinery industry, medical device industry, as well as aerospace and defense industry.

\section{ACKNOWLEDGEMENTS}

The authors gratefully acknowledge the German research agencies DLR (project 01IC 10L 01C) and DFG (project Ku 619/19-2).

\section{REFERENCES}

Arnold, D., H. Isermann, A. Kuhn, H. Tempelmeier and K. Furmans. 2008. Handbuch Logistik. Springer Verlag. Berlin.

Bockholt, F., W. Raabe and M. Toth,. 2011. "Logistic Assistance Systems for Collaborative Supply Chain Planning”. Int. Journal Simulation and Process Modelling 6(4):297-307.

Boysen, D., M. Fliedner and A. Scholl. 2007. "Production planning of mixed-model assembly lines. Overview and extensions". Jena research papers in business and economics, 06/2007. http://nbnresolving.de/urn:nbn:de:gbv:27-20100728-101738-6 [Accessed July 16, 2013].

Cohen, S., R. Geissbauer, A. Bhandari, and M. D’heur. 2008 “Global Supply Chain Trends 2008-2010”. PRTM Management Consultants.

Deiseroth, J., D. Weibels, M. Toth, and A. Wagenitz. 2008. "Simulationsbasiertes Assistenzsystem für die Disposition von globalen Lieferketten - Simulation-based Decision Support System for the Disposition of Global Supply Chains". In Advances in Simulation for Production and Logistics Applications, Edited by M. Rabe, 41-50. Stuttgart: Fraunhofer IRB Verlag.

Farkisch, K. 2011. Data-Warehouse-Systeme kompakt. Aufbau, Architektur, Grundfunktionen. Springer Verlag. Berlin.

Geissbauer, R. and M. D’heur. 2008. “Global Supply Chain Trends 2010-2012”. PRTM Management Consultants.

Herlyn, W. J. 1990. „Zur Problematik der Abbildung variantenreicher Erzeugnisse in der Automobilindustrie“. Ph.D. thesis. VDI. Düsseldorf.

Institute for Environment and Sustainability 2010. ELCD core database version II. European Commission. http://lca.jrc.ec.europa.eu/lcainfohub/datasetArea.vm [Accessed January 09, 2012].

Klingebiel, K., B. Hellingrath, S. Pires, and L. F. Scavarda. 2011. "Integrated Design and Evaluation of Logistic Networks: Oriented Placement of the Order Penetration Point". Brazilian Journal of Operations \& Production Management Vol 8:89-102. 
Kuhn, A., Hellingrath, B. and J. Hinrichs. 2008. "Logistische Assistenzsysteme". In Software in der Logistik. Weltweit sichere Supply Chains, 20-26. München: huss-Verlag.

Meyr, H. 2004. "Supply chain planning in the German automotive industry". OR Spectrum 26:447-470.

Motta, M., A. Wagenitz, B. Hellingrath, and R. Weller. 2008. "Gestaltung logistischer Netzwerke - ein Praxisbericht - Design of Logistic Networks - A Case Study". In Advances in Simulation for Production and Logistics Applications, Edited by M. Rabe, 21-30. Stuttgart: Fraunhofer IRB Verlag.

Odette 2004. "Demand Capacity Planning". Odette International Limited. London.

Toth, M., A. Wagenitz. 2009. „Neue Wege für die effektive Planung logistischer Netzwerke. Dynamische Verfügbarkeitsplanung mit Hilfe von Assistenzsystemen“. Industrie Management 25(2). 55-58. Berlin: GITO Verlag.

Toth, M., A. Wagenitz, K. Klingebiel. 2011. "Logistic Assistance Systems for Effective Supply Chain Planning"

Wagenitz, A. 2007. „Modellierungsmethode zur Auftragsabwicklung in der Automobilindustrie“. Ph.D. thesis, Technical University Dortmund. Germany. http://www.ub.tu-dortmund.de/Eldorado [Accessed June 14, 2013].

VDI (Verband Deutscher Ingenieure) 1992. VDI 3633: Simulation von Logistik-, Materialfluß- und Produktionssystemen, Düsseldorf.

\section{AUTHOR BIOGRAPHY}

KLAUS LIEBLER is a project manager at Fraunhofer IML working in the Department Supply Chain Engineering since 2008 focussing his activities in the field of automotive production planning. In May 2013 he has finished his PhD thesis. His e-mail address is klaus.liebler@iml.fraunhofer.de.

ULRIKE BEISSERT joined the Fraunhofer IML, Department Supply Chain Engineering as a research associate in 2011, focussing her activities primary on simulation-based planning of material flows, storage and transport concepts. Before she worked as a research associate at the Bauhaus-University Weimar, where she received her $\mathrm{PhD}$ from Faculty Civil Engineering introducing a constraint-based simulation concept for the scheduling of Construction Processes. Her e-mail address is ulrike.beissert@iml.fraunhofer.de.

MARCO MOTTA is the head of Department Supply Chain Engineering since 2013. Before he worked 9 years as project manager and teamleader in the institute focussing his activites on automotive industry. Since 2009 Marco Motta is executive director of the LogProIT GmbH. His e-mail address is marco.motta@iml.fraunhofer.de.

AXEL WAGENITZ is a Professor of Information Systems at Hamburg University of Applied Sciences. He worked for 16 years at the Fraunhofer IML and, amongst others, promoted in that time the development of OTD-Net.His e-mail address is axel.wagenitz@iml.fraunhoder.de. 